\title{
Duplication Images in Vascular Sonography
}

\author{
Jonathan M. Rubin, $M D$, PhD, Jing Gao, MD, \\ Keith Hetel, MD, Robert Min, MD
}

\begin{abstract}
Objective. The purpose of this study was to determine the characteristics and phenomena of duplication (or mirror) images in vascular sonography. Methods. We retrospectively reviewed 5 cases of vascular sonography that produced duplication images performed between June 30, 2009, and January 10,2010. The 5 vascular studies included the subclavian artery (2), subclavian vein (1), carotid artery (1), and abdominal aorta (1). The characteristics of the color flow images and spectral Doppler waveforms in the artifactual vessels and in the true vessels were analyzed. Sources and mechanisms of producing duplication images were assessed. Results. We were able to generate color flows in the artifactual vessels that were the same as in the true vessels in 2 cases. However, we were able to generate images in which the apparent flow directions in the artifactual vessels differed from the actual flow observed in the true vessels in all 5 cases by changing the orientation of the ultrasound beam relative to the true flow and the reflecting surface. Conclusions. Duplication images result from mirror reflections producing multipath artifacts. The appearance of the flow in an artifactual vessel on color Doppler imaging strongly depends on the angle of the incident sound beam and the surface structure of the strong reflector relative to the flow in the true vessel. Alternating the scanning plane and the angle of the incident sound beam to the reflector may help distinguish a duplication artifact from a true vessel in ambiguous cases. Key words: carotid artery; color Doppler image; duplication image; incident sound beam; mirror artifact; multipath artifact; reflector; subclavian artery.
\end{abstract}

Received May 18, 2010, from the Department of Radiology, University Hospital, University of Michigan, Ann Arbor, Michigan USA (J.M.R.); and Department of Radiology, New York-Presbyterian Hospital, Weill Cornell Medical College, New York, New York USA (J.G., K.H., R.M.). Revision requested June 7, 2010. Revised manuscript accepted for publication June 10, 2010.

Address correspondence to Jonathan M. Rubin, MD, PhD, Department of Radiology, University Hospital, University of Michigan, 1500 E Medical Center Dr, Ann Arbor, MI 48109-5030 USA.

E-mail: jrubin@med.umich.edu

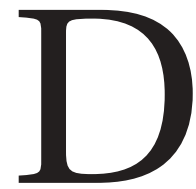

uplication images (or mirror images) are commonly observed in ultrasound scanning. They have been reported on gray scale, ${ }^{1-4}$ color flow, and spectral Doppler imaging. ${ }^{5-11}$ The mechanism of ultrasound energy generating the duplication image is complex, and it is based on the combination of the physical properties of ultrasound and time-depth misregistration by the ultrasound scanner. ${ }^{1,3,7} \mathrm{~A}$ multipath artifact is produced when the incident sound beam propagates to a mirrorlike strong reflector that induces multiple reflections in the visualized objects, which then ultimately propagate back to the transducer. It takes longer for multiple reflected echoes to return to the transducer than it takes for the first reflection to return. As a result, incorrect time assumptions are made by ultrasound scanners, which then display the late-arriving echoes at locations deeper than the reflectors' true locations. These incorrectly positioned echoes can, in fact, be imaged on the opposite side of the strong reflector, pro- 
ducing an artifactual repeat appearance of the primary object in the wrong location. ${ }^{8,9}$

The theory of producing duplication images in vascular sonography is more complicated than that in gray scale imaging because blood flow information is presented over and above the gray scale images as color flow and spectral Doppler imaging. Some literature suggests that in vascular duplication images, the flow direction, velocity, and signal strength in the artifactual (mirrored) vessel should be comparable or nearly identical to those in the true vessel..$^{5,6}$ However, we have clinically observed that this may not be the case, and others have suggested that the source and artifactual vessels may not have the same flow properties. ${ }^{10,11}$ For instance, both the artifactual and true vessels may have the same flow direction on some images but opposite directions on the others. To address these issues, we analyzed the characteristics of color flow images and spectral Doppler waveforms in 5 cases with duplication images to assess possible sources generating variable appearances of color flow and spectral waveforms in the artifactual vessels.

\section{Materials and Methods}

We retrospectively reviewed 5 cases of vascular sonography that produced duplication images performed between June 30, 2009, and January 10,2010 . The 5 vascular studies included the subclavian artery (2), subclavian vein (1), carotid artery (1), and abdominal aorta (1). The reasons for requiring vascular sonography included evaluating arterial stenosis, screening for an abdominal aortic aneurysm, and detecting subclavian vein thrombosis. All patients $(2$ men and 3 women; age range, 30-83 years; mean age, 67 years) were referred by physicians in the Departments of Emergency Medicine and Vascular Surgery at the New York-Presbyterian Hospital of Weill Cornell Medical College.

An Acuson Sequoia 512 system (Siemens Medical Solutions, Mountain View, CA) was used to scan the carotid artery, subclavian artery, and subclavian vein with a $6-\mathrm{MHz}$ linear array transducer, while the abdominal aorta was scanned with a 3.5- to 5-MHz curved linear array transducer. There was no special preparation before sonography. The patients were all scanned in the supine position. Imaging was started with gray scale and supplemented with color flow and spectral Doppler imaging on longitudinal and transverse planes of the vessels. All of the artifactual vessels were observed behind totally reflecting targets such as gas in lungs or bone. All images were stored in the Department of Radiology's picture archiving and communications system, and the review was made on static images only.

There was no consent form needed because this was a retrospective study. The Institutional Review Board at Weill Cornell Medical College approved the study (approval number 1002010878). The study was Health Insurance Portability and Accountability Act compliant.

\section{Results}

Of the 5 cases, 2 were duplication artifacts of the subclavian artery, the long axis of which was nearly parallel to the orientation of the interface of the lung apex. Scanning parallel to the long axis produced antegrade flow in both the artifactual and the true subclavian arteries with Doppler angle correction by steering the color box (Figure 1, A-C). However, when the scan plane was rotated from the long axis to the near short axis, the flow in the artifact reversed compared to the true vessel. The artifactual artery developed flow away from the scan head, while the true subclavian artery had flow toward the scan head (Figure 1D). Moreover, the direction of the artifactual flow differed from the true flow on color flow imaging in the short axis in the remaining 3 cases, including the subclavian vein (Figure 2), carotid artery (Figure 3), and abdominal aorta (not shown).

\section{Discussion}

Sonography relies on physical assumptions to assign the location of each received echo. ${ }^{1}$ To generate duplication images, in general, there are at least 3 factors required: (1) the incident sound beam propagates to a mirrorlike strong reflector with a sufficient difference in acoustic impedance from the tissue more proximal to the transducer to create multiple reflections; (2) multiple reflections (multipath artifact) from the mirror back into the flow stream produce an 
additional Doppler shift; and (3) multiple reflections are eventually backscattered to the ultrasound transducer along the reverberation path, which are erroneously displayed deep to the original vessel. In addition to these factors, the angle between the direction of blood flow and the incident sound beam plays a major role in how the flow direction and velocity are displayed in the duplication image.

The artifactual vessels could be misinterpreted as a true additional vessel, arterial wall dissec- tion, or possibly an ulcerated plaque ${ }^{8,9}$ if the phenomenon of the duplication image is not understood. Furthermore, the additional complication that the color flow imaging and spectral Doppler waveform in the artifactual vessel may vary from the true flow makes interpretation even more difficult.

It is already known that the artifactual vessel can have flow properties similar to those of the real source vessel, and it has been shown that the flow in artifactual vessel can differ from the orig-

Figure 1. A, Long-axis color flow imaging in which a subclavian artery duplication is observed at the lung apex (green arrow), which acts as a strong reflector. The strong reflector has the same orientation as that of the true subclavian artery, and it is located between the true artery and the artifactual artery (white arrow). The flow toward the transducer is presented in both the true subclavian artery and the artifactual artery as red. The color box is steered to angle the incident sound beam as nearly parallel to the true flow as possible. B, Spectral Doppler imaging with angle correction $\left(49^{\circ}\right)$ shows that the flow in the true subclavian artery (white arrow) in the long axis is toward the transducer. Antegrade flow is displayed above the baseline on the spectrum. $\mathbf{C}$, Antegrade flow toward the transducer in the artifactual artery documented with spectral Doppler imaging at the same Doppler angle of $49^{\circ}$. The velocity and spectral waveforms in the artifactual artery (white arrow) are similar to those in the true artery (B). D, Same subclavian artery being scanned in short-axis color flow imaging. There is enough angulation so that the true flow's Doppler component is largely toward the transducer (red). The flow direction in the artifactual artery (white arrow) is largely away from the transducer (blue). The artifactual artery is located on the opposite side of the lung apex (green arrow) from the true subclavian artery.

A

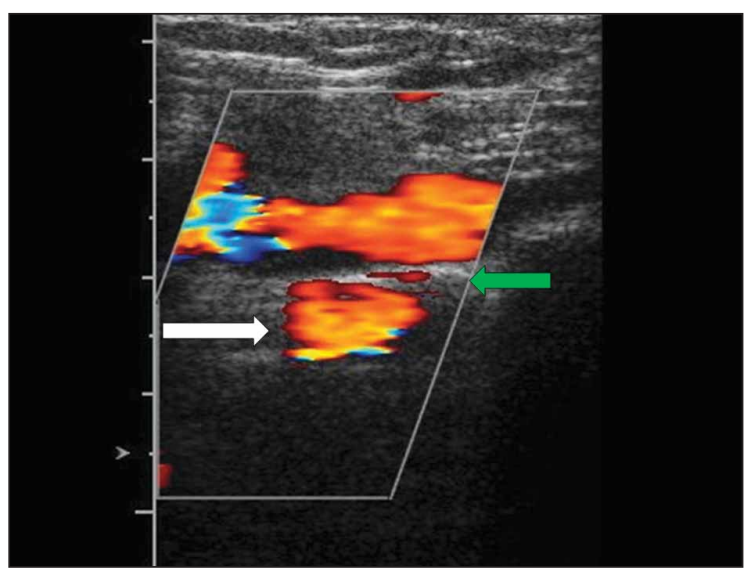

c

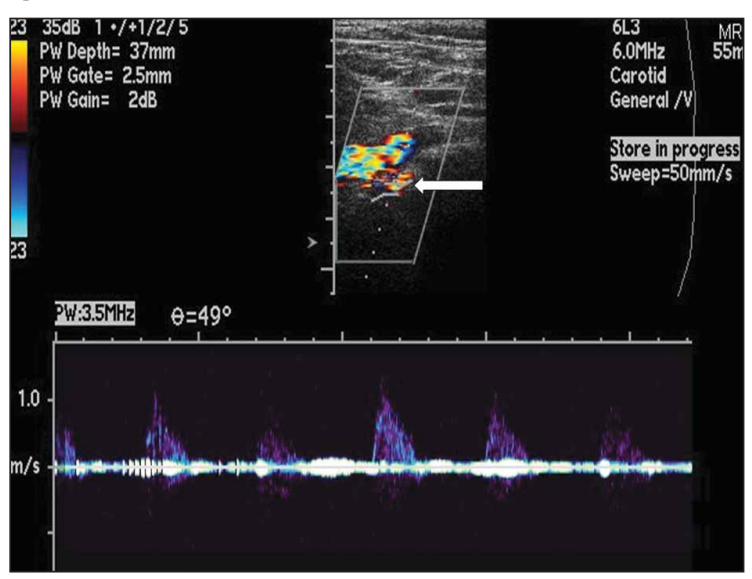

B

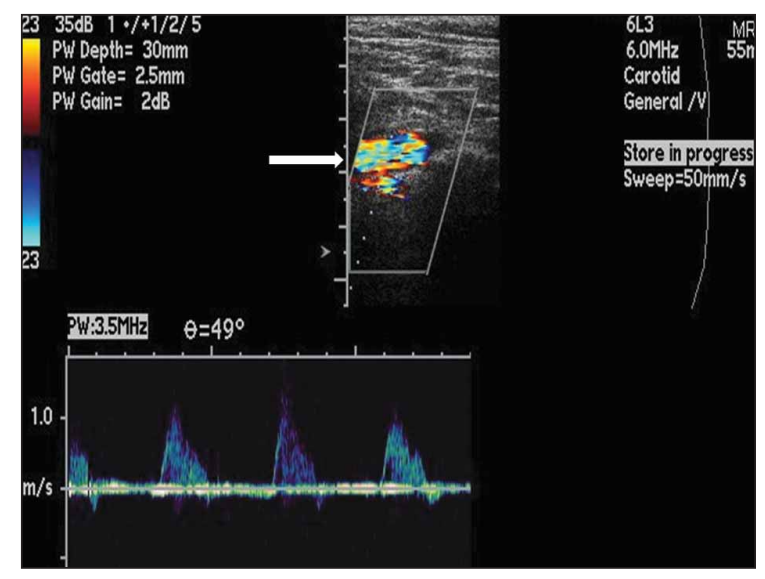

D

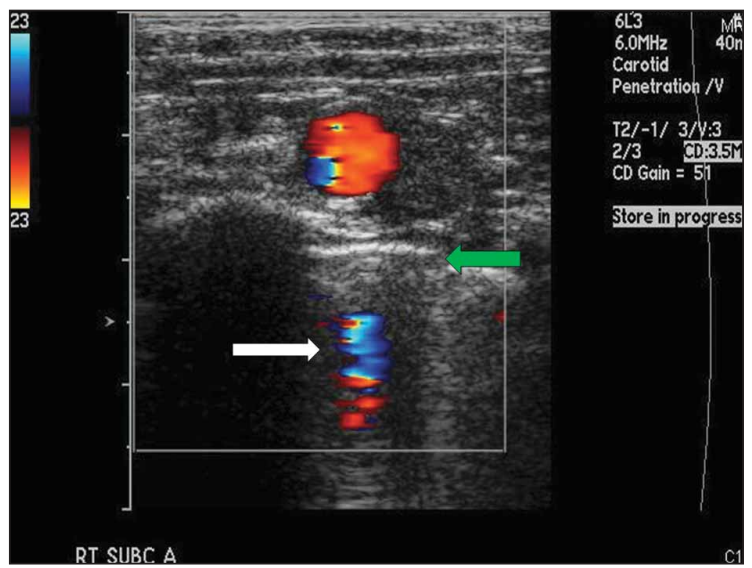




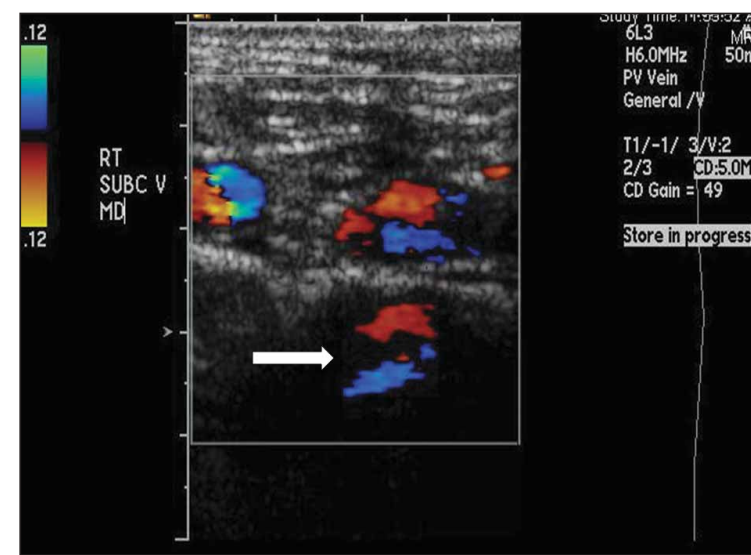

Figure 2. Duplication image on a transverse view of the subclavian vein. The artifactual and true flows are reversed relative to the mirror. The flow in the artifactual vein (arrow) is blue/red compared with the flow in the actual vein (red/blue). The color separation results from swirling flow in which flow directions in the different components of the flow are reversed in the true vessel and the duplicated vessel. MD indicates mid; RT, right; and SUBC V, subclavian vein.

inal vessel. ${ }^{10,11}$ However, we have shown that the apparent flow direction in the artifactual vessel can vary substantially from the true flow (Figures $1 \mathrm{D}, 2$, and $3 \mathrm{~A}$ ) and can actually vary in any given case depending on the orientations of the transducer to the true vessel and its flow. The appearance of the flow in an artifactual vessel on color flow imaging strongly depends on the angle of the incident sound beam, the surface structure of the strong/mirror reflector, and the angle of the sound beam reflected off the mirror relative to the flow in the true vessel.

The flow direction and velocity in the artifactual vessel would be the same as the true flow if the angle of the multipath reflection produces a second Doppler angle relative to the flow stream that is equal to that of the original beam. This is likely to happen if the actual flow in the true vessel is nearly parallel to the surface of the strong reflector and the flow pattern in the source vessel is stable across the distance between the original beam and the multipath beam (Figure 4A).

The flow direction in the artifactual vessel begins to differ when the Doppler angle from the multipath beam is different from that of the original beam (Figure 4B). This can be most dramatic when making a near transverse image of a vessel, so the reflected beam off the mirror has a Doppler angle that is approximately $180^{\circ}$ from the original (Figure 4C). This would create an artifact with reverse flow. Depending on the relative angle of the mirror to the true flow, almost any Doppler shift could, in theory, be generated in the mirror vessel, including a mirror produced by a vessel that is out of plane.

Figure 3. A, Duplication image generated in a transverse color flow image of the carotid bulb. The incident sound beam is nearly perpendicular to the arterial wall and reflector. Antegrade flow is seen in the true carotid bulb (green arrow), and retrograde flow is visualized in the artifactual carotid bulb (white arrow). B, Spectral Doppler imaging confirms that the flow direction in the artifactual carotid bulb differs from that in the true carotid bulb. A slow recording rate allows a single spectral Doppler trace to be displayed from both the true and artifactual carotid bulbs on one image. The spectral Doppler waveform is inverted on the image. CCA indicates common carotid artery; and LT, left.

A

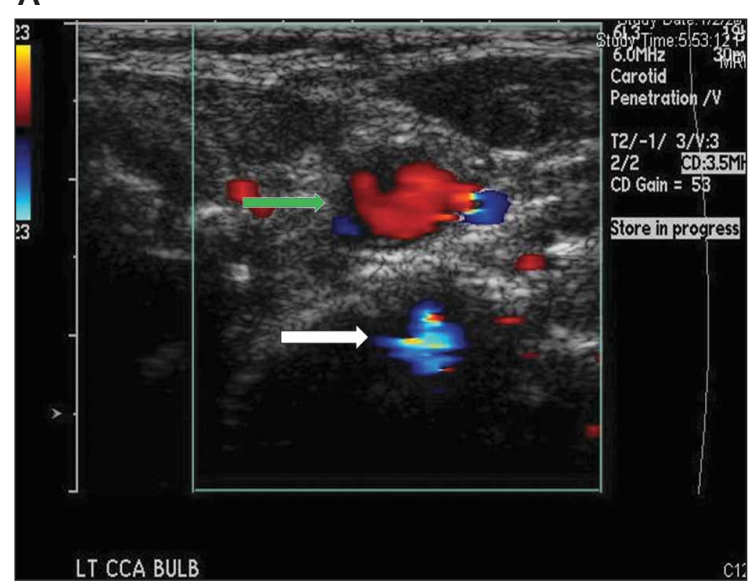

B

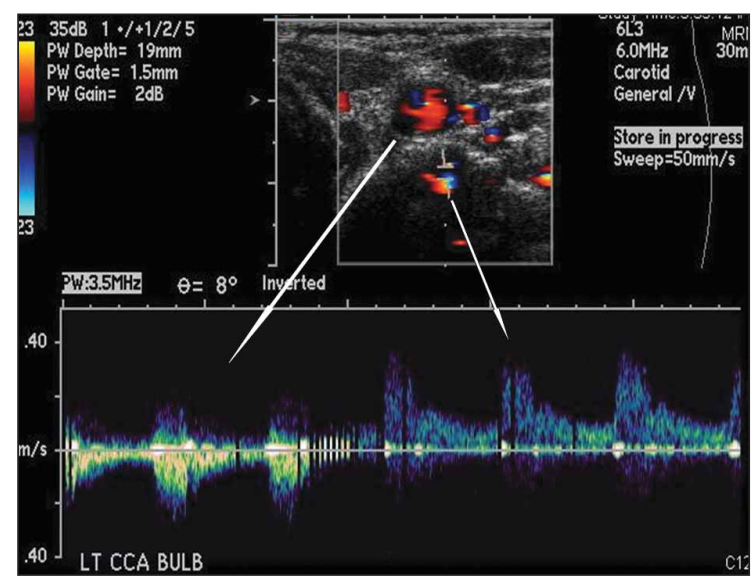


In conclusion, in vascular duplication images, the direction of artifactual flow displayed in color flow and spectral Doppler imaging is not necessarily the same as the actual flow. The depicted flow in the duplicated image strongly depends on the relationship of the incident sound beam, the true flow direction, and the geographic surface of the strong reflector. Alternating the scan- ning plane and the angle of the incident sound beam to the strong reflector, which may be accomplished by changing the orientation of the transducer, adjusting the Doppler angle in spectral Doppler imaging, and/or steering the color box in color flow imaging, may help distinguish a duplication artifact from a true vessel in ambiguous cases.

A

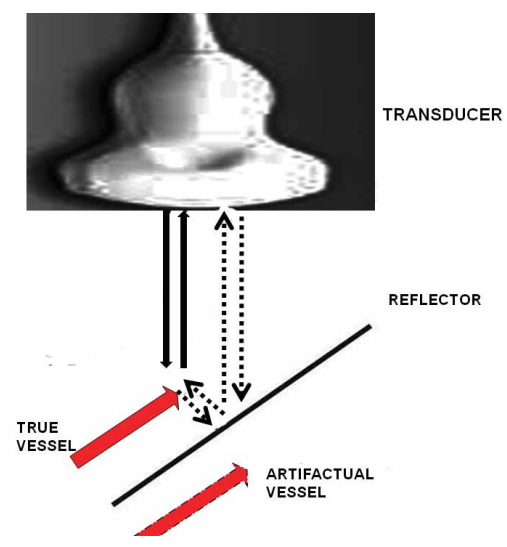

Figure 4. A, This illustration is used to explain how the same flow direction in both the true vessel and the artifactual vessel in a vascular duplication artifact can occur. The angle of the multipath reflection (dotted black arrows) produces a second Doppler angle that is equal to that of the original beam (solid black arrows), only the multipath reflection is entering the flow stream from the opposite side of the vessel. The actual flow in the true vessel (top red arrow) is nearly parallel to the surface of the strong reflector, and the flow pattern in the true vessel is stable across the distance between the original beam and the multipath beam. Thus, the flow stream looks the same for the original beam and the reflected beam. B, This illustration shows how reverse flow in the artifactual vessel (blue arrow) can be created relative to the true vessel (red arrow). The solid black arrows represent the sound beams producing the true Doppler image, and the dotted black arrows represent the sound beams producing the artifactual Doppler image. The Doppler angle relative to the flow stream from the multipath beam is greater than $90^{\circ}$, causing the flow to appear away from the transducer (represented by the direction of the blue arrow), while the Doppler angle from the true beam is less than $90^{\circ}$, so the flow appears toward the transducer (represented by the direction of the red arrow). C, This illustration shows how a transverse scan of a vessel with a slight elevational tilt of the transducer will produce a mirror artifactual vessel that has reverse flow to the true vessel. The image of the vessel is an ellipse to show that this is not a perpendicular cross section. There must be some tilt to produce a Doppler shift. The tilt is represented by the elliptical appearance of the vessel. The dotted black arrows in the true vessel indicate that the direction of flow is toward the transducer, and the true vessel is shown as red. The initial sound beam (solid black arrows) enters the true vessel and is reflected from red blood cells in the vessel. Some of the sound passes through the true vessel, hits the reflector, which in this case is curved like the anterior surface of a vertebral body, and is reflected back into the vessel. This multipath sound is depicted by the orange arrows. However, now the flow is away from the sound source, which is the front surface of the reflector. The Doppler angle is rotated by $180^{\circ}$, ie, opposite to the Doppler angle of the original beam, which produces a negative Doppler shift, so that the flow in the artifactual vessel (blue) is opposite that in the true vessel. 


\section{Duplication Images in Vascular Sonography}

\section{References}

1. Feldman MK, Katyal S, Blackwood MS. US artifacts. Radiographics 2009; 29:1179-1189.

2. Scanlan KA. Sonographic artifacts and their origins. AJR Am J Roentgenol 1991; 156:1267-1272.

3. Mann GS, Robinson AJ, LeBlanc JG, Heran MK. Abdominal aortic pseudomass in a child: a diagnostic red herring. J Ultrasound Med 2008; 27:307-310.

4. Keogh CF, Cooperberg PL. Is it real or is it an artifact. Ultrasound Q 2001; 17:201-210.

5. Reading CC, Charbonaeu JW, Allison JW, Cooperberg PL. Color and spectral Doppler mirror-image artifact of the subclavian artery. Radiology 1990; 174:41-42.

6. Gustavson S, Olin JW. Images in vascular medicine: mirror image artifact. Vasc Med 2006; 11:175-176.

7. Hebbard PD. Artifactual mirrored subclavian artery on ultrasound imaging for supraclavicular block. Can J Anaesth 2009; 56:537-540.

8. Arning C, Eckert B. The diagnostic relevance of colour Doppler artifacts in carotid artery examinations. Eur J Radiol 2004; 51:246-251.

9. Arning C. Mirror image artifacts of color Doppler images causing misinterpretation in carotid artery stenoses. J Ultrasound Med 1998; 17:683-686.

10. Pozniak MA, Zagzebski JA, Scanlan KA. Spectral and color Doppler artifacts. Radiographics 1992; 12:35-44.

11. Ferrara K, DeAngelis G. Color flow mapping. Ultrasound Med Biol 1997; 23:321-345. 